\title{
Development of New Measurement System of Errors in the Multiaxial Machine Tool for an Active Compensation
}

\author{
Noureddine Barka and Abderrazak El Ouafi \\ Mathematics, Computer and Engineering Department, University of Quebec at Rimouski, Rimouski, QC, Canada G5L 3A1 \\ Correspondence should be addressed to Noureddine Barka; noureddine_barka@uqar.ca
}

Received 16 September 2016; Accepted 20 November 2016

Academic Editor: Christian Kirchsteiger

Copyright (C) 2016 N. Barka and A. El Ouafi. This is an open access article distributed under the Creative Commons Attribution License, which permits unrestricted use, distribution, and reproduction in any medium, provided the original work is properly cited.

Error compensation techniques have been widely applied to improve multiaxis machine accuracy. However, due to the lack of reliable instrumentation for direct and overall measurements, all the compensation methods are based on offline measurements of each error component separately. The results of these measurements are static in nature and can only reflect the conditions at the moment of measurement. These results are not representative under real working conditions because of disturbances from load deformations, thermal distortions, and dynamic perturbations. This present approach involves the development of a new measurement system capable of dynamically evaluating the errors according to the six degrees of freedom. The developed system allows the generation of useful data that cover all machine states regardless of the operating conditions. The obtained measurements can be used to evaluate the performance of the machine, calibration, and real time compensation of errors. This system is able to perform dynamic measurements reflecting the global accuracy of the machine tool without a long and expensive analysis of various error sources contribution. Finally, the system exhibits compatible metrological characteristics with high precision applications.

\section{Introduction}

The contribution of machine tools in the development of several industrial fields is demonstrated. However, during recent decades, the economic environment has forced the industry to new standards of performance in terms of quality, productivity, costs, and production time. These market constraints have evolved much faster than the performance of existing machines. The technology behind these machines is based on concepts dating back several decades and is almost to the limit of their potential. This relative stagnation is explained by the fact that technological developments in this sector are more motivated by the needs of users than by the manufacturer's initiatives. This situation highlights the need to develop strategies to respond to these new challenges by improving the performance of the machines and making them evolved to a higher level of technology [1-3].

The loss of precision in the machine tools is due to the geometrical imperfections of the mechanical structure of the machine or follows the modification of this structure under static, thermal, and dynamic stresses. Even if some of these imperfections can be reduced by the improvement of machine design, it is nevertheless difficult to eliminate completely their effects. The technological limitations and the costs associated with this solution led to the introduction of the concept of errors compensation [4-8]. However, the compensation techniques such as they are practised imply the need for measuring, under typical operating and environmental conditions, all the components of the error, before combining them through mathematical models for then generating the data necessary to make the correction in real time $[9,10]$. Such a step, although it makes possible the correction of a great proportion of the systematic error, does not make it possible to evaluate the random components of the error. Moreover, several other limitations largely compromise the potential of this approach. Among these limitations are (i) the importance of the deadlines and the costs necessary for the remote measurement of the errors under various operating conditions; (ii) the difficulty of covering all sources of errors being able to have effects on the precision of the machine; 
(iii) the need for carrying out the periodic calibration of the machine whose geometry undergoes continuous variations because of wear, tiredness, and other static and dynamic effects; (iv) the errors being evaluated under static conditions, with the variations they undergo under dynamic conditions not being taken into account; (v) the errors being measured individually and the mechanisms of interaction between the various sources of errors not considered. To circumvent these limitations, it becomes necessary to develop a dynamic and integrated approach of measurement able to evaluate, in real time, the individual components of the errors associated with the geometrical, thermal, and dynamic effects. This step makes it possible to provide a reliable representation of the machine state for robust and effective adaptive compensation [11-16].

The objective of this paper is to develop a measurement system in real time to identify simultaneously all components of the volumetric error in the multiaxial machine tools. Indeed, the most recent advances in instrumentation have made possible the development of techniques for measuring performance both in terms of accuracy and in terms of speed and cost. The prototype is able to monitor and quantify the components of the error generated when moving along a main axis. Based on an optoelectronics structure, the proposed approach makes it possible to evaluate simultaneously the errors according to 5 of the 6 degrees of freedom of each machine head, regardless of the operating conditions. Moreover, this approach makes it possible to simplify in a substantial way the procedures of calibration and metrological performance evaluation of the machine tools. Compared with reference measurement techniques, this approach presents some significant advantages in terms of precision and in terms of manipulation speed and cost.

\section{Measurement System Configuration}

Recent advances in the instrumentation field made possible the development of new, powerful measurement techniques. The measurement approach, suggested during this research, is based partly on these innovations, combining the optical and electronic components to carry out a high precision system of measurement. A diagram of the device of measurement is presented in Figure 1. The basic configuration is made up of two parts: a fixed plate constituting the measurement reference and a mobile plate directly attached to the moved mechanism. The fixed part supports a He-Ne laser source, three semireflective mirrors, and three photodetectors. The moving part comprises a retroreflector and 3 flat mirrors. The reference beam generated by the $\mathrm{Ne}-\mathrm{He}$ laser source is divided into 3 distinct beams. The first one is projected on flat mirror $\mathrm{FM}_{1}$ and then reflected to photodetector $\mathrm{PD}_{1}$. The second beam is projected on retroreflector RR and then directed to photodetector $\mathrm{PD}_{2}$. The third beam is reflected to photodetector $\mathrm{PD}_{3}$ using 2 flat mirrors $\mathrm{FM}_{2}$ and $\mathrm{FM}_{3}$. Any deviation of the mobile plate compared to the reference would cause variations of beam position on the photodetectors. These variations are obtained starting from the output signals of the photodetectors connected to an electronic circuit for

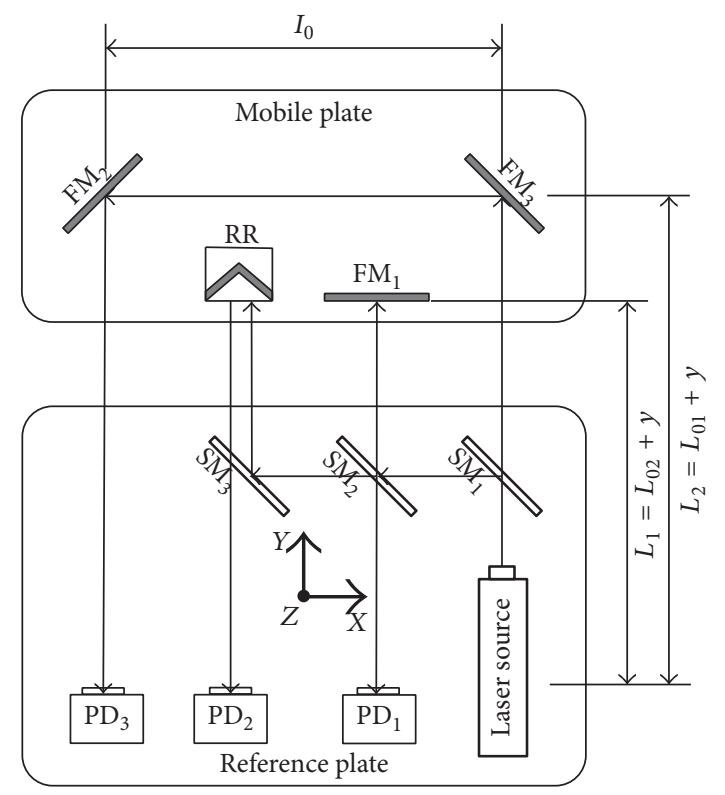

FIGURE 1: Disposition of measurement system components.

conditioning and preprocessing. A computer is used for acquisition, treatment, and exploitation of various signals.

The He-Ne laser presents low-cost solution with good repeatability. The separators of the nonpolarized laser beam prevent polarization of the incident beam. Their low absorption allows these dielectric spacers to separate the beams by $50 \%$. They are made from a substrate in high precision, having a thermal expansion factor of the order of $7.10^{-6 \circ} \mathrm{C}^{-1}$, to minimize the distortion of the wavelength. Moreover, the inclination of the substrate avoids any possible back reflection to the laser. The position sensors are made of silicon photodiodes in a single crystal with a quadruple electrode. Compatible with low-power laser beams and other infrared beams, they allow obtaining accurate information on the linear positioning of the beam in both horizontal and vertical directions.

It is important to note the degrees of freedom principle. In a linear constraint, displacement along a known axis leads to the elimination of 5 of the 6 degrees of freedom of the moving part compared to the guidance support. The movement in the direction of the remaining degree of freedom is controlled with precision using a suitable control device. A linear displacement of the moving part along a specific axis involves deviations according to three directions: three translations (positioning and straightness errors) and three rotations (yaw, pitch, and roll). Consequently, the three beams obtained from the principal beam must be aligned in the direction of the moving axis so that the considered beams are received by the photodetectors. The principle and the method of calculation of the position of each beam on a photodetector are presented in Figure 2. The beam position is calculated according to the photocurrents from the voltages $V_{A}, V_{B}, V_{C}$, and $V_{D}$. The positions in the horizontal $\left(P_{H}\right)$ and 


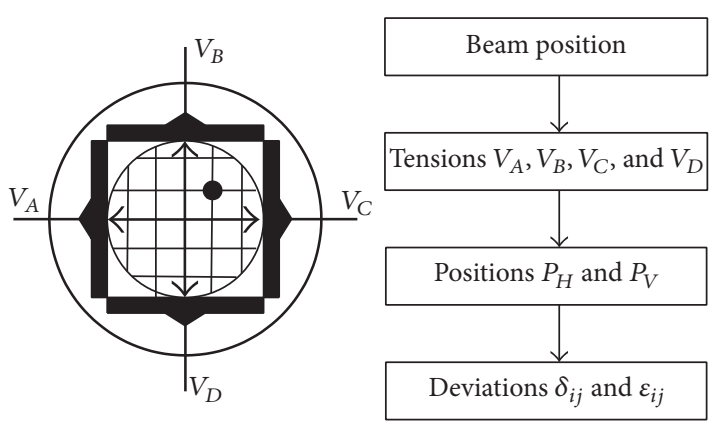

FIGURE 2: Calculation of beam position.

vertical $\left(P_{V}\right)$ planes are calculated starting from these exits according to preestablished relations

$$
\begin{aligned}
& P_{H}=\left(\frac{V_{A}-V_{C}}{V_{A}+V_{C}}\right) \\
& P_{V}=\left(\frac{V_{B}-V_{D}}{V_{B}+V_{D}}\right) .
\end{aligned}
$$

The photodetector $\mathrm{PD}_{1}$ receives the beam reflected by flat mirror $\mathrm{FM}_{1}$, with its positions $\left(P_{H 1}, P_{V 1}\right)$ sensitive to yaw and pitch, respectively. These readings are not affected by the linear position of the mirror. The positions $\left(P_{\mathrm{H} 2}, P_{V 2}\right)$ of the photodetector $\mathrm{PD}_{2}$, which receives the beam reflected by the retroreflector $\mathrm{RR}$, are sensitive to the straightness errors in the horizontal and vertical plans. The beam position is reflected by 2 flat mirrors $\mathrm{FM}_{2}$ and $\mathrm{FM}_{3}$ (oriented at $45^{\circ}$ ) and represented by the positions $\left(P_{H 3}, P_{V 3}\right)$ on the photodetector $\mathrm{PD}_{3}$. This constitutes the cumulative effects of the angular errors (pitch and roll) as well as the straightness in the horizontal plane. Based on the dimensional and geometrical configuration of the measurement system and interactions between data from the photodetectors, some arithmetic relations were established to calculate the various errors. Thus, for each relative movement between the two plates (mobile and references plates), six coordinates are available to evaluate the five components of error. Redundant measurement can be used as checking criterion. In the case of the machine tool, measurements obtained in this manner reflect the real position of the tool related to the part and make it possible to evaluate the deviations that it undergoes compared to its programmed trajectory. These deviations can be caused by geometrical, static, thermal, and dynamic sources. Once the $P_{H}$ and $P_{V}$ positions are calculated for $\mathrm{PD}_{2}$, the deviation in the horizontal plan is regarded as a horizontal straightness error while any deviation in the vertical plan is considered a vertical straightness error

$$
\begin{aligned}
& \delta_{y x}=P_{H 2} \\
& \delta_{y z}=P_{V 2} .
\end{aligned}
$$

The two angular components $\varepsilon_{y x}$ and $\varepsilon_{y y}$ depend on the rotation around the two horizontal and vertical axes. Any

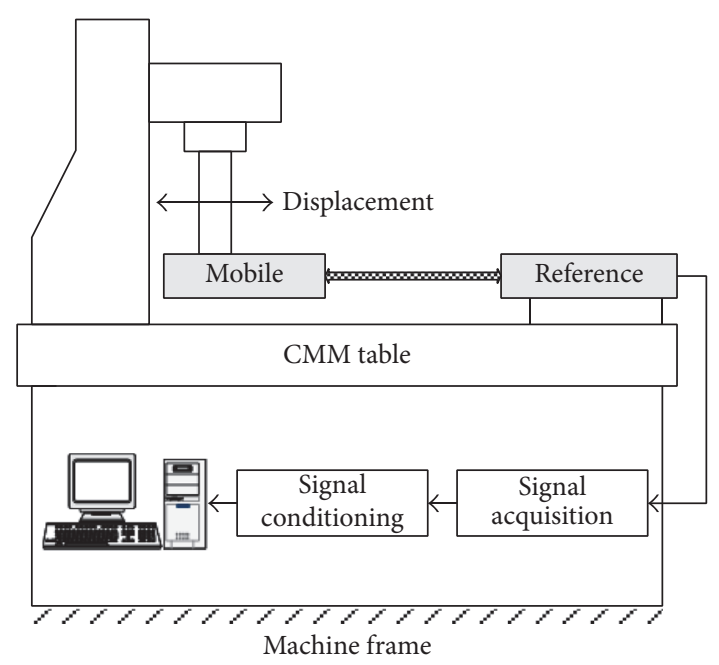

FIGURE 3: Schematic representation of the experimental setup.

rotation of the target with respect to the $x$-axis and the $z$ axis will be translated into yaw and pitch using trigonometric relationships

$$
\begin{aligned}
& \varepsilon_{y x}=\tan ^{-1}\left(\frac{P_{H 1}}{L_{02}+y}\right) \\
& \varepsilon_{y z}=\tan ^{-1}\left(\frac{P_{V 1}}{L_{02}+y}\right) .
\end{aligned}
$$

The roll error $\varepsilon_{y y}$ depends on the rotation about the $y$ - and $z$-axes. The angle of rotation about the $y$-axis, which is considered the axis of movement, is given by the trigonometric relationship

$$
\varepsilon_{y y}=\tan ^{-1}\left(\frac{P_{V 3}}{l_{0}}-\frac{P_{V 1}}{L_{01}+y}\right) .
$$

\section{Experimental Setup and Preliminary Tests}

With the aim of analyzing the responses of the new measurement system in order to determine its metrological characteristics, a coordinate measurement machine (CMM) is used to carry out evaluation tests. The diagrammatic representation of the experimental setup is illustrated in Figure 3. The overall system includes both the mobile and reference plates supporting the optical accessories. The fixed part of the measurement device is placed on the machine marble, whereas the moving part is fixed on the CMM head.

Preliminary tests were carried out in order to optimize the utilisation conditions of the measurement system. In fact, it seems important to maximize the precision and the stability before starting the measurement validation. The obtained results show clearly that environmental conditions (light, vibrations, draught, and moisture) have a negative influence on the quality of the system responses. To evaluate the impact of these phenomena, an analysis of sensitivity was performed. This study made it possible to determine their actions and the mechanisms of their interactions and 


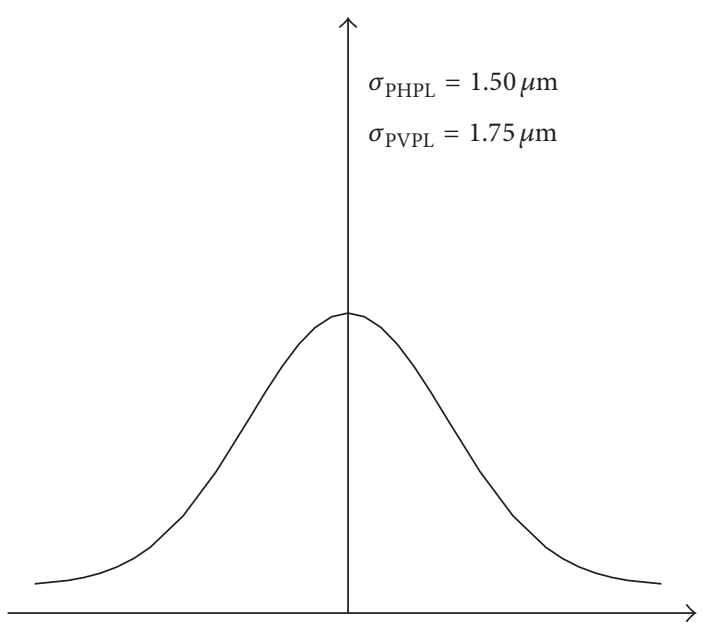

(a)

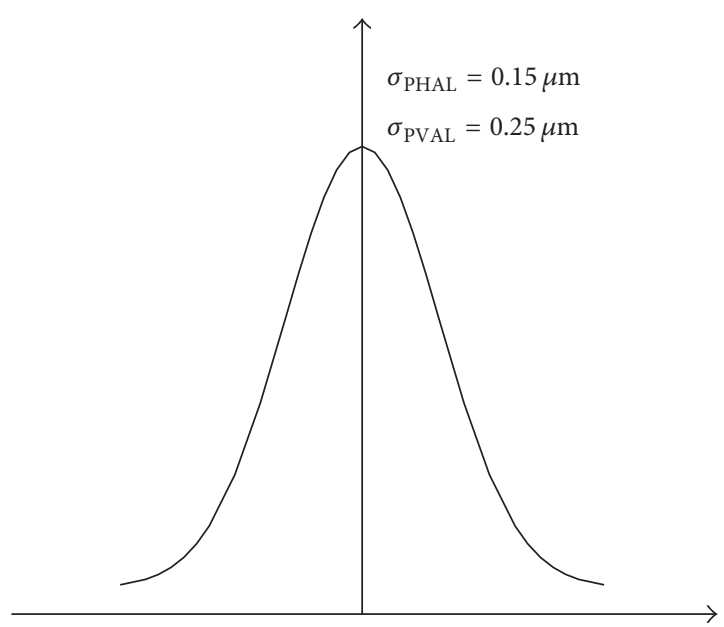

(b)

FIGURE 4: Responses of photodetectors based on different conditions.

to release the conditions allowing the reduction of their effects. Thus, the two parts of the measurement system were covered so as to reduce the effects of the external sources of light. The use of the covers made it possible to improve substantially the stability and the luminosity of the beams. Figure 4 illustrates the improvements obtained and shows, that with this arrangement, the variation of the signals decreases from $1.50 \mu \mathrm{m}$ to $0.15 \mu \mathrm{m}$ relative to the horizontal plan and from $1.75 \mu \mathrm{m}$ to $0.25 \mu \mathrm{m}$ relative to the vertical plan. The use of filters also made it possible to reduce the noise and the adverse effects due to the variations of the other surrounding conditions. Moreover, with an aim of improving the repeatability of the system and of taking account of the effects heating, a 100-minute preheating period was observed before each test series (Figure 5).

To calibrate the system and to reduce the effect of environmental conditions, artificial neural networks present an interesting solution. In fact, models based artificial neural networks (ANN) are commonly used to compensate for the effects of temperature and relative humidity. The integration of these models in the compensation is easy and simple to implement. However, the model cannot be generalized for all surfaces of the position detector, because of nonlinearity due to the geometrical shape of the position detector. To overcome these limitations, it is necessary to calibrate the position sensor by developing an algorithm to estimate the horizontal and vertical positions while correcting for nonlinearity due to the concave shape of the surface position sensors. This algorithm must take into account the position of the beam on the projection surface from the position detector by combining all of the environmental factors. To get a rough estimation of the resolution readings, calibration tests were performed. During these tests, the mobile plate is mounted on the displacement member of the CMM related to the fixed plate so as to bring the laser beam to the center of each photodetector position. This position is then the reference position for position sensor calibration. A scan of the entire surface of the position detector is made to generate the

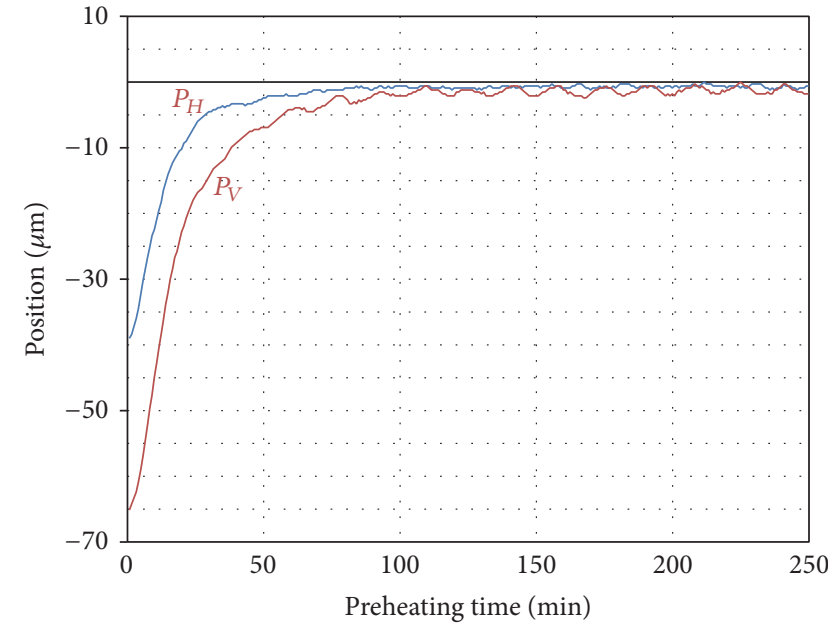

FIgURE 5: Preheating curve of laser source.

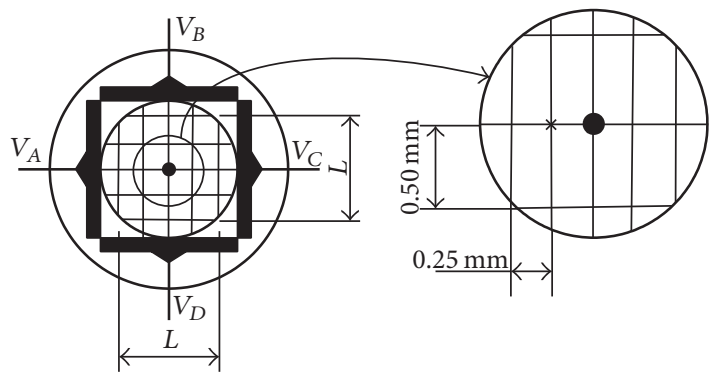

Figure 6: Calibration data pattern.

necessary data for modeling. The pattern of data acquisition is shown in Figure 6.

The positions $P_{V}$ and $P_{H}$ of the laser beam on the photodetector are estimated in accordance with the voltages $V_{A}, V_{B}, V_{C}$, and $V_{D}$. The model consists of a representation of 


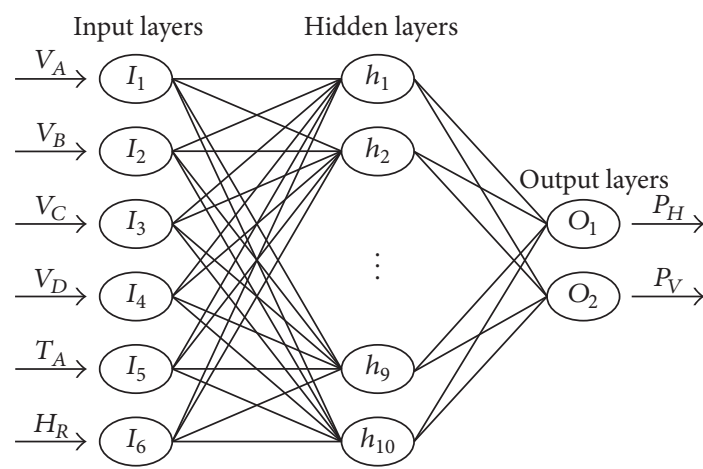

FIGURE 7: ANN model architecture of positions prediction.

the positions behavior based on voltages and the influence of ambient temperature $\left(T_{A}\right)$ and relative humidity $\left(H_{R}\right)$. The modelling process is performed by the ANN technique multilayer perceptron (MLP) known by its good forecast ability, simplicity, and flexibility of prediction. The MLP consists of three layers; the first and the third layers have the same number of neurons compared to the inputs and the outputs, respectively. The hidden layer is developed with taking into consideration the input and the output neurons and the complexity of the estimated parameters to avoid the overfitting. Several training attempts are important to determinate the 10 neurons of the hidden layer that met the best accuracy. An artificial neural controller capable of making appropriate corrections is developed to compensate for the effects of environmental conditions. Note that, to validate the effectiveness of the model (Figure 7), it is necessary to verify the model using new data that were not used during the training process. Tests on the coordinate measuring machine have led to the generation of useful data for training and for validation.

Figures 8 and 9 show the residual error of $P_{H}$ and $P_{V}$ positions during training and validations stages. During the training phase, the mean square errors (MSE) are, respectively, of the order of $76.15 \mu \mathrm{m}$ and about $92.81 \mu \mathrm{m}$ for the $P_{H}$ and $P_{V}$ positions. The variance is in the range of $0.601 \mu \mathrm{m}^{2}$ for $P_{H}$ position and about $0.650 \mu \mathrm{m}^{2}$ for the $P_{V}$ position. During the validation phase, the two mean square errors (MSE) are, respectively, of the order of $277.71 \mu \mathrm{m}$ and $240.27 \mu \mathrm{m}$ for the $P_{H}$ and $P_{V}$ positions. These errors are 3 times higher compared to training errors. The variance is of the order of $1.797 \mu \mathrm{m}^{2}$ for the $P_{H}$ position and about $1.795 \mu \mathrm{m}^{2}$ for the $P_{V}$ position. The residual errors have a relatively large variation with respect to that of the training phase. The results show that the data taken in the peripheral region of the photodetector detector have a time uncertainty larger than that measured, which corresponds exactly to the specifications given by the manufacturer of the optical element. To reduce the uncertainty, it is important to operate as close as possible to the central area by improving the total alignment of the measurement system (Table 1).

The analysis model was used to evaluate the influence of temperature on the position. On average, a temperature variation of about $\pm 0.5^{\circ} \mathrm{C}$ causes a change in the beams

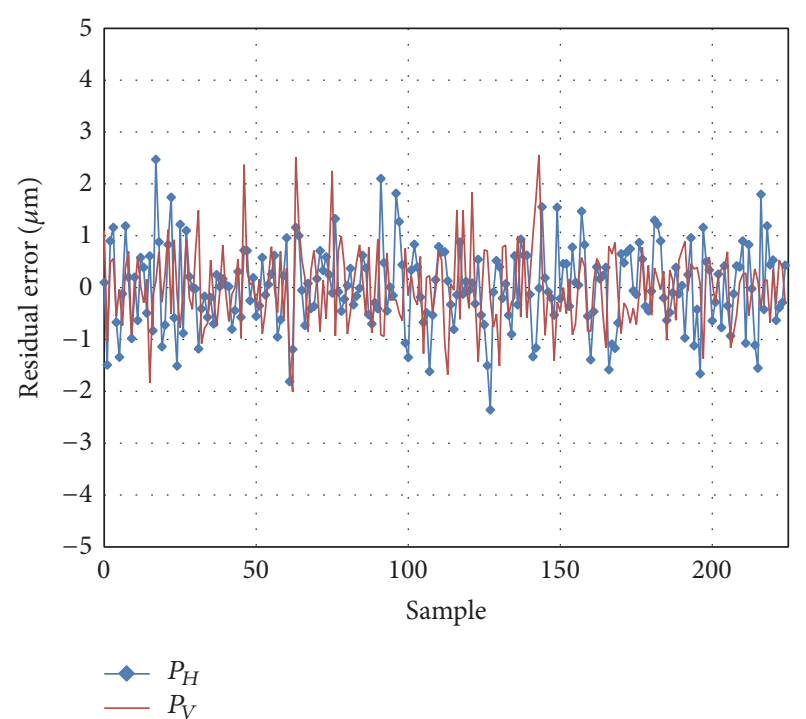

FIGURE 8: Residual error of training stage.

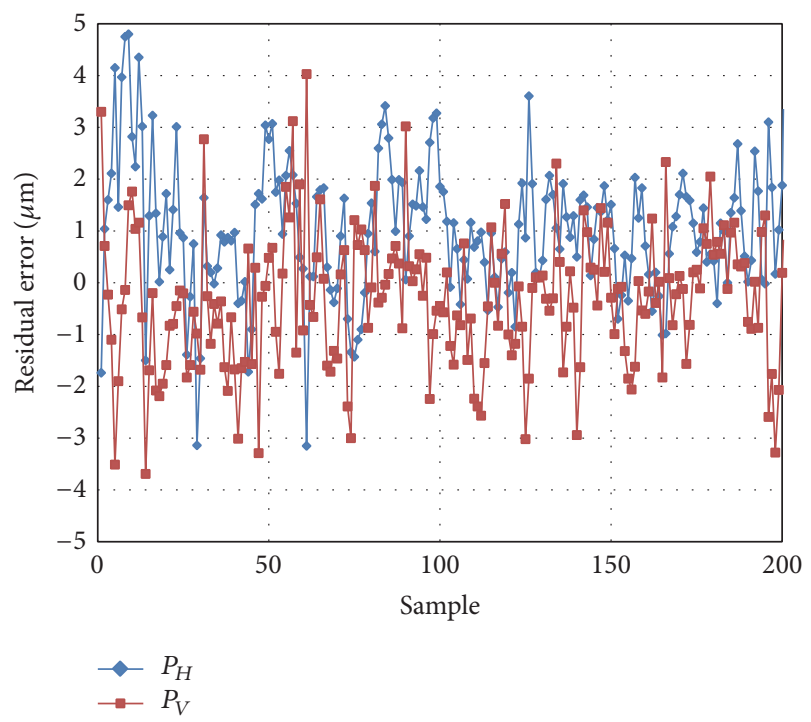

FIGURE 9: Residual error of validation stage.

position of $1 \mu \mathrm{m}$ in the horizontal direction and $1.5 \mu \mathrm{m}$ in the vertical direction. However, the variation due to temperature is not linear, because it depends on the laser beam position on the photodetector surface and the uncertainty associated with the calibration of temperature sensor integrated into the experimental setup. Table 2 shows the influence of ambient temperature on the laser beam position. Also, it is possible to evaluate the effect of the relative humidity (RH) on the measurements. Consequently, each average $\mathrm{RH}$ variation of $0.5 \% \mathrm{HR}$ position causes an average variation of $0.15 \mu \mathrm{m}$ in the horizontal direction and $0.20 \mu \mathrm{m}$ in the vertical direction of the beam position on the photodetector (Table 3). Following the impact assessment of the environmental conditions on the laser beam, the sensitivity analysis consists of quantifying the sensitivity of the signal under these disturbing factors. The resulting models help to estimate the 
TABLE 1: Comparison of training and validation performances.

\begin{tabular}{lcc}
\hline & Training & Validation \\
\hline $\operatorname{MSE}\left(P_{H}-\mu \mathrm{m}\right)$ & 76.15 & 277.71 \\
$\operatorname{MSE}\left(P_{V}-\mu \mathrm{m}\right)$ & 92.81 & 240.27 \\
Variance $\left(P_{H}-\mu \mathrm{m}^{2}\right)$ & 0.601 & 1.797 \\
Variance $\left(P_{V}-\mu \mathrm{m}^{2}\right)$ & 0.650 & 1.795 \\
\hline
\end{tabular}

TABLE 2: Influence of temperature on the positions.

\begin{tabular}{lcc}
\hline & Horizontal position $\left(P_{H}\right)$ & Vertical position $\left(P_{V}\right)$ \\
\hline $\pm 0.5^{\circ} \mathrm{C}$ & $1 \mu \mathrm{m}$ & $1.5 \mu \mathrm{m}$ \\
$\pm 1^{\circ} \mathrm{C}$ & $3.25 \mu \mathrm{m}$ & $5 \mu \mathrm{m}$ \\
$\pm 2^{\circ} \mathrm{C}$ & $5 \mu \mathrm{m}$ & $9 \mu \mathrm{m}$ \\
\hline
\end{tabular}

TABLE 3: Effect of relative humidity (RH) on the positions.

\begin{tabular}{lcc}
\hline & Horizontal position $\left(P_{H}\right)$ & Vertical position $\left(P_{V}\right)$ \\
\hline $\pm 0.5 \% \mathrm{RH}$ & $0.15 \mu \mathrm{m}$ & $0.20 \mu \mathrm{m}$ \\
$\pm 1 \% \mathrm{RH}$ & $0.45 \mu \mathrm{m}$ & $0.65 \mu \mathrm{m}$ \\
$\pm 2 \% \mathrm{RH}$ & $0.75 \mu \mathrm{m}$ & $1.10 \mu \mathrm{m}$ \\
\hline
\end{tabular}

influence of different sources of error. A neural compensator was necessary to reduce variations due to environmental conditions, taking into account the whole area of the detector. The stability has been improved due to the quick action of the compensator. The effort has controlled variations resulting from various sources, both systematic and random in nature. It remains to achieve metrological characterization of the new measurement system in order to determine its accuracy and uncertainty related to the operation and validate its performance using a multiaxial machine tool.

\section{Experimental Results}

The last phase of this project is to evaluate the metrological characteristics of the new measurement system (NMS) and validate their performances using a multiaxial machine tool by comparison with a precision interferometer laser (Renishaw-ML10). A protocol for the referencing and installation of the measuring system on the machine is built up to facilitate the alignment procedures and reduce setup time. To get a good estimation of the 5 error components along the main displacement axis, a laser interferometer (ML10) with an accuracy of about $0.1 \mu \mathrm{m}$ and thermal stability on the order of $\pm 0.0011 \mathrm{ppm}$ was used to evaluate the measurements obtained by the NMS. Since the ML10 does not allow the measurement of more than one component at the same time, the adopted measurement procedure is to compare the NMS to the ML10 for each error component. In each test, a series displacement (front-back) was conducted to validate the linear (horizontal and vertical straightness) and angular deviations (yaw and pitch). With regard to the roll, there is no direct laser ML10 configuration to validate it. The calibration of the NMS generates algorithms to provide a reliable and effective measurement by reducing errors due to the total alignment of the NMS and systematic errors due

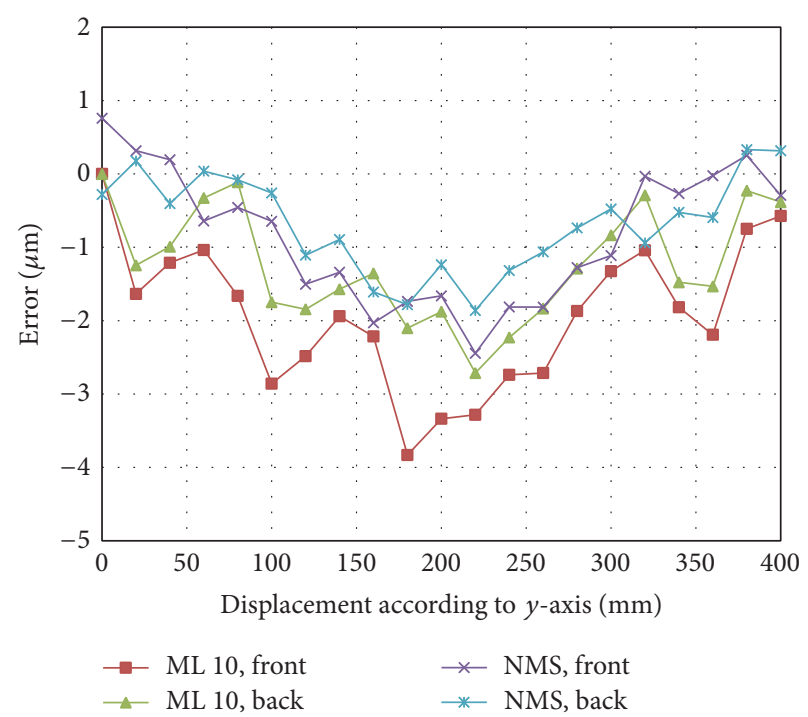

FIGURE 10: Measurement of horizontal straightness.

to the measurement system itself. This calibration must be repeated after every system installation on a new machine. A reset reading is required to define an absolute NMS to return to its initial position. The developed models are used to estimate the errors according to the beam positions on the photodetectors and the distance traveled by the mobile plate.

The validation tests are conducted on the CMM. The reference plate of the NMS is placed on the machine frame, while the mobile plate is fixed on the machine displacement head. The NMS is aligned according to an alignment protocol. Since the interferometer laser uses an elementary measurement approach, it cannot, however, validate more than one error component at each test. It is necessary to mount in parallel with the NMS while respecting the proposed configurations for each measure.

Figure 10 shows the result of the horizontal straightness measuring using both approaches. The NMS calibration is performed using a linear model which transforms data related to the horizontal position from the photodetector for horizontal position straightness. This calibration is to eliminate systematic errors and cosine errors that can affect the entire measurement system. Both curves (front and back) obtained by the ML10 laser have the same tendency, and they exhibit an offset in the form of mechanical hysteresis behavior, primarily reflecting geometrical errors and the guiding bridge CMM relative to the $y$-axis. The maximum straightness error is about $3.8 \mu \mathrm{m}$. The two curves (return) obtained by NMS have the same tendency as reference curves. The total straightness error is on the order of $2.6 \mu \mathrm{m}$. Regarding the back curve, the encoder receives an offset on the order of $0.25 \mu \mathrm{m}$ which is due to the mechanical hysteresis and the hysteresis CMM measuring system itself.

Figure 11 shows the result of vertical straightness measuring using both approaches. The NMS is calibrated using a linear model which transforms data of deviations in the vertical position. In this case, both curves (front and back) obtained using the ML10 laser have the same tendency 


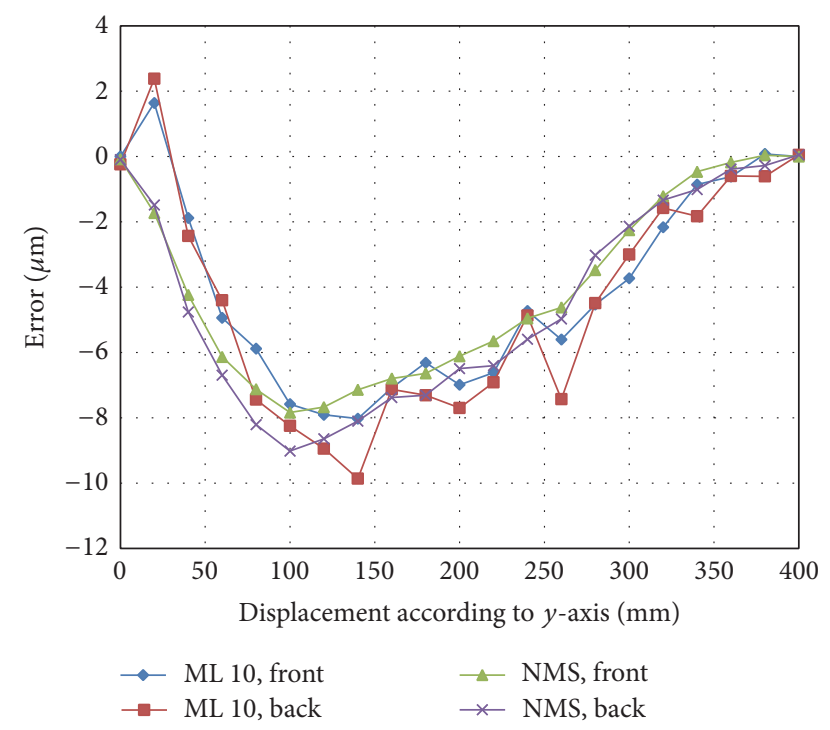

Figure 11: Measurement of vertical straightness.

and they exhibit an offset in the mechanical hysteresis. The maximum vertical straightness error is about $9.64 \mu \mathrm{m}$. The curves obtained by NMS have the same tendency as the reference curves. The maximum vertical straightness error is on the order of $9.01 \mu \mathrm{m}$. Regarding the back curve, the encoder receives an offset on the order of $0.15 \mu \mathrm{m}$ which is due to the mechanical hysteresis of the CMM and the hysteresis of the system measurement itself.

The statistical analysis performed was used to compare the correlation between the two curves (front and back). A comparative study of the performance curves according to the displacement cycle is conducted. Regarding the sequence "front" the residual error between the curves obtained with the two techniques is on the order of $11.130 \mu \mathrm{m}$ for the horizontal straightness about $13.665 \mu \mathrm{m}$ for the vertical straightness. As for the sequence "back" the residual error is two times less than the cycle "front" for the horizontal straightness and about $9.371 \mu \mathrm{m}$ for vertical straightness. Analysis of the variance of the difference between the two curves (front and back) reveals a variation nearly equal to the vertical and horizontal straightness. This analysis was used to predict a rough estimation of the overall accuracy of the new measurement system based on the variance. The measurements obtained by the NMS are introduced through a calibration model to overcome the cosine and systematic errors as well as the correction of the basic equation used to calculate the yaw and pitch errors. This calibration takes into account the actual position in both horizontal and vertical directions as well as the distance traveled by the mobile plate.

Figure 12 illustrates the extent of the yaw using both approaches in a specific front and back cycle. The error evolution is nearly linear depending on the $y$-axis. The maximum error is about 5.9 arcsec. The results obtained by NMS are consistent with those taken by the ML10. The two curves (front and back) have the same tendency and they vary in a linear style as a function of displacement. However, there is an offset of about 0.45 arcsec caused by the hysteresis

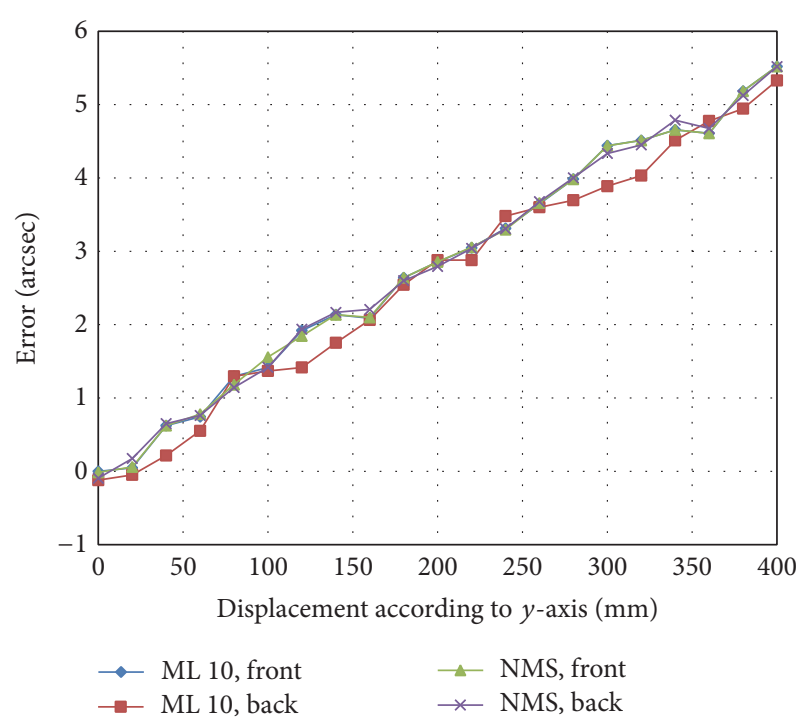

FigURE 12: Measurement of yaw.

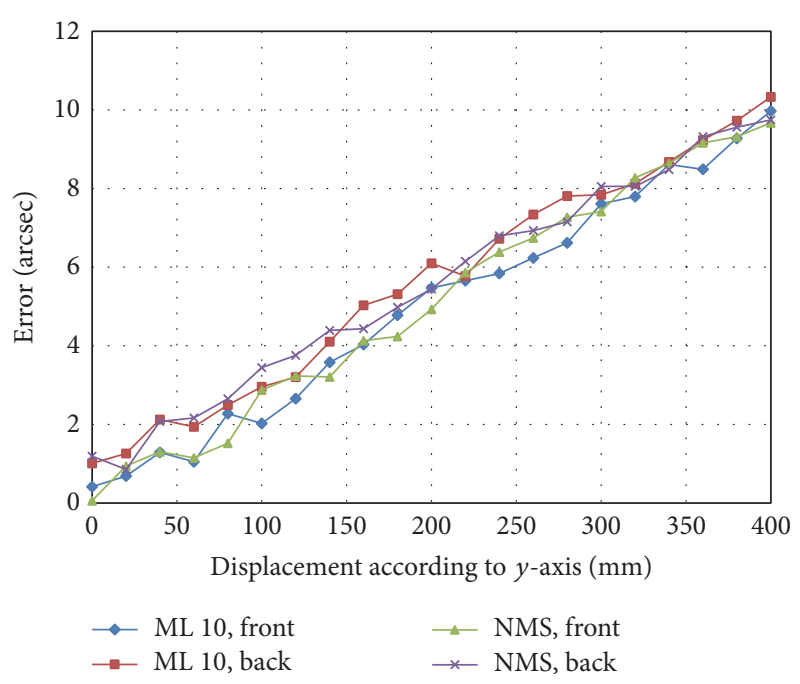

FigURE 13: Measurement of pitch.

between the NMS and the CMM. The maximum yaw error measured by the NMS, at about 5.9 arcsec, is comparable to that obtained by the laser ML10.

Figure 13 illustrates the pitch measurement by using the two approaches in a front and back cycle. The curves obtained have the same trend as for the ML10 laser measurement. Unlike for the straightness measurements, the hysteresis error is minimal. The maximum error is bigger than the error of lace and is of the order of 9.456 arcsec. The results obtained by NMS are consistent with the measures taken by the ML10. However, there is offset of about 0.25 arcsec caused by the hysteresis between the NMS and the CMM. The maximum error is comparable to that obtained by the ML10 which is of the order of 9.474 arcsec.

It is also interesting to compare the two approaches by calculating the mean square error (MSE) and variance. Concerning the yaw, the variance is more pronounced between 


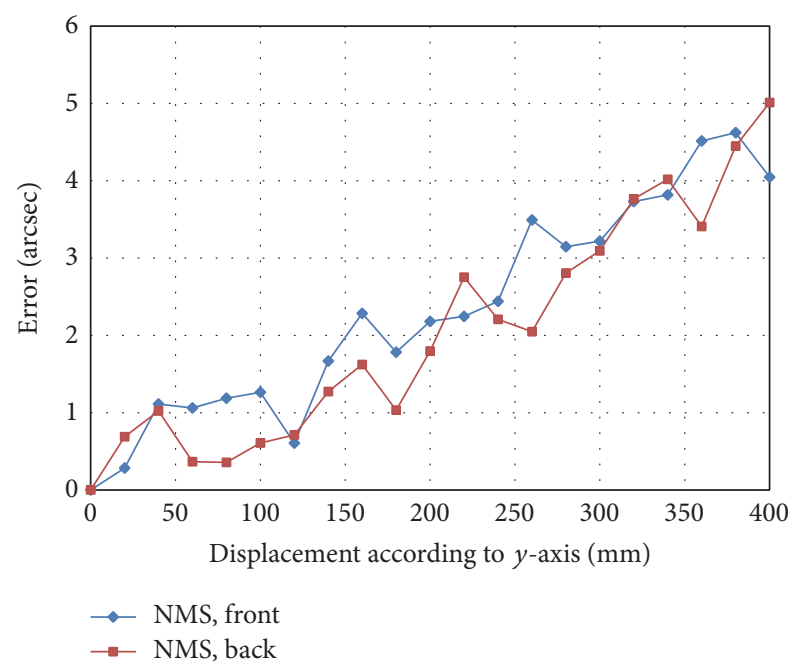

FigURE 14: Measurement of roll.

the two curves than between the front and back cycles. The MSE is about 0.331 arcsec for the front cycle and on the order of 1.204 arcsec for the back cycle. The variance is around 0.016 arcsec and 0.045 arcsec for the two cycles, respectively. As for pitch, the MSE are comparable and are of the order of 0.151 arcsec and 0.170 arcsec for the two cycles, respectively. The variances are comparable to the variances of the measures taken by the ML10.

The roll measurement is illustrated in Figure 14. The error calculation is performed by applying the relationship determined below. The roll error is less significant than the other angular errors. The validation of this measure is still necessary to get an idea of the accuracy of the NMS. However, the error validation requires the establishment of special and accurate, structural validation. Both curves have the same trend and the maximum error is about 5 arcsec. It is important to note that the ML10 did not allow the measurement of the roll error.

\section{Conclusions}

The new measurement system consists of an optoelectronics structure able to measure simultaneously and dynamically 5 of the 6 components of the error of displacement. This system enables the identification and the compensation of error in real time regardless of the operating conditions. The tests carried out show that the precision of the system is advantageously comparable with that obtained by recognized measurement techniques. In addition to its precision, the system of measurement proposed is also characterized by its speed, its facility of use, and its cost. Moreover, by its dynamic character, it makes it possible to integrate geometrical, thermal, and dynamic effects and to include them in the same measurement operation. The NMS is developed and designed for the simultaneous and direct evaluation of five geometrical errors on a multiaxial machine tool using a combination of various optical and electronic components. The optimal arrangement of these elements in the existing system was used to measure the positions of the laser beam on the position sensors and convert these positions into real errors. This configuration allows the quantification of linear deviations or horizontal straightness and vertical straightness and three angular errors, yaw, pitch, and roll.

The new measurement system required a sensitivity analysis to study the behavior over time of the signals from the three position sensors. This analysis helped to reveal the impact of environmental conditions on the measurements. Although it helped to stabilize variations in a temperature range between $17^{\circ} \mathrm{C}$ and $25^{\circ} \mathrm{C}$, it is not possible to remedy the convex nature of photodetector. The model was developed to characterize the horizontal and vertical positions of the beam according to each parameter's influence. The developed model helps to calculate the horizontal position $\left(P_{H}\right)$ and the vertical position $\left(P_{V}\right)$ as a function of the voltages from the detector $\left(V_{A}, V_{B}, V_{C}\right.$, and $\left.V_{D}\right)$, the temperature, and relative humidity.

The characterization of the metrological parameters of the NMS was needed to determine operating conditions and characteristics in terms of accuracy and resolution. The statistical analysis was used to study the accuracy and fidelity in different points. In addition, tests performed on the CMM were used to validate the performances of the new measurement system using a precision laser (ML10). The developed measurement system is characterized by its speed, accuracy (about $\pm 1.5 \mu \mathrm{m}$ ), and ease of use. The accuracy could be improved by adopting a more comprehensive and detailed analysis of environmental conditions and operating conditions. In addition, the dynamic conditions must be identified in order to further integrate them into the final model. The full integration of the measurement system in machine tools requires optimization of prototypes in terms of size, components, and protection from environmental disturbances.

\section{Competing Interests}

The authors declare that they have no competing interests.

\section{References}

[1] M. S. Burdekin, "Cutting tests for accuracy assessment," in Technology of Machine Tools, vol. 5, pp. 9-11, 1980.

[2] J. B. Bryan, "A simple method for testing measuring machines and machine tools part 1: principles and applications," Precision Engineering, vol. 4, no. 2, pp. 61-69, 1982.

[3] Y. Kakino, Y. Ihara, Y. Nakatsu, and K. Okamura, “The measurement of motion errors of NC machine tools and diagnosis of their origins by using telescoping magnetic ball bar method," CIRP Annals-Manufacturing Technology, vol. 36, no. 1, pp. 377380, 1987.

[4] J. Tlusty and F. Koenigsberger, "Concepts of machine tools accuracy," Annals of CIRP, vol. 24, 1975.

[5] W. Knapp and E. Matthias, "Test of the three-dimensional uncertainty of machine tools and measuring machines and its relation to the machine errors," CIRP Annals-Manufacturing Technology, vol. 32, no. 1, pp. 459-464, 1983.

[6] W. Knapp, "Circular test for three-coordinate measuring machines and machine tools," Precision Engineering, vol. 5, no. 3, pp. 115-124, 1983. 
[7] F. W. Jones, Performance Evaluation of Precision Numerically Controlled Turning Equipment, MTDR, 1970.

[8] J. Jedrzejewski, W. Kwasny, D. Milejski, and M. Szafarczyk, "Selected diagnostic methods for machine tools acceptance tests," CIRP Annals-Manufacturing Technology, vol. 34, no. 1, pp. 343-346, 1985.

[9] M. S. Burdekin, "Instrumentation and techniques for the evaluation of machine tool errors, machine tool accuracy," Technology of Machine Tools, vol. 5, pp. 9-17, 1980.

[10] T. Treib, A New Measuring System for Testing the Geometric Accuracy of Linear Guideways, IWF (ETHZ), Zuerich, Switzerland, 1988.

[11] Y. Kagawa, S. Matsumiya, K. Yamazaki, and Y. Yang, Laser-CCD Based Sensor System for Real Time Detection of Motion Linearity, Department of Mechanical and Aeronautical engineering, University of California, 2003.

[12] M. A. Donmez, D. S. Blomquist, R. J. Hocken, C. R. Liu, and M. M. Barash, "A general methodology for machine tool accuracy enhancement by error compensation," Precision Engineering, vol. 8, no. 4, pp. 187-196, 1986.

[13] V. Ragunath, Thermal Effects on the Accuracy of Numerically Machine Tools, Purdue University, Indianapolis, Ind, USA, 1985.

[14] A. H. Slocum, Precision Machine Design, Prentice-Hall, Englewood Cliffs, NJ, USA, 1992.

[15] S. Sartori, P. C. Cresto, M. Di Ciommo, and T. K. Kancheva, "A way to improve the accuracy of a co-ordinate measuring machine," Measurement, vol. 6, no. 2, pp. 50-54, 1988.

[16] K. Busch, H. Kunzmann, and F. Waeldele, "Numerical errorcorrection of a coordinate measuring machine," in Proceedings of the International Symposium on Metrology for Quality Control in Production, Tokyo, Japan, 1984. 


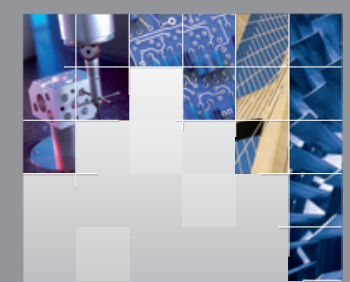

\section{Enfincering}
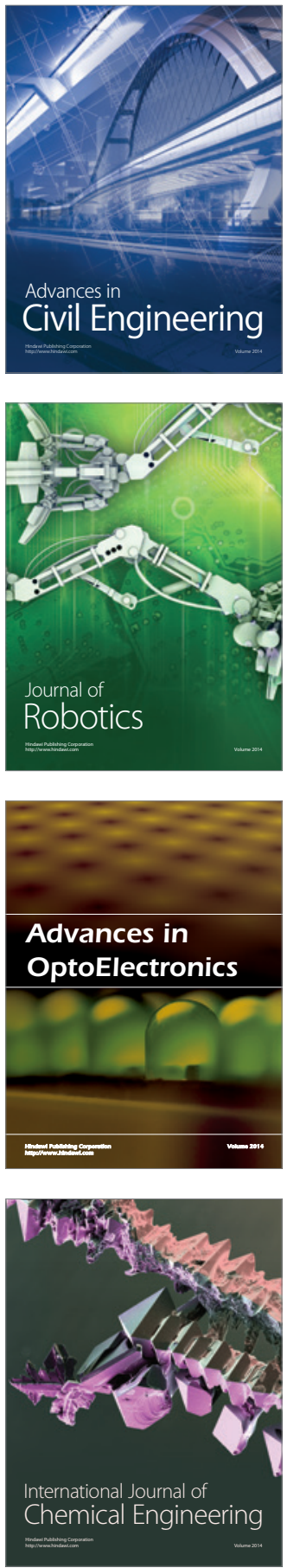

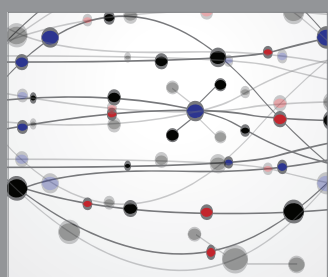

The Scientific World Journal

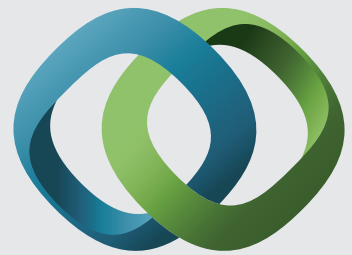

\section{Hindawi}

Submit your manuscripts at

http://www.hindawi.com
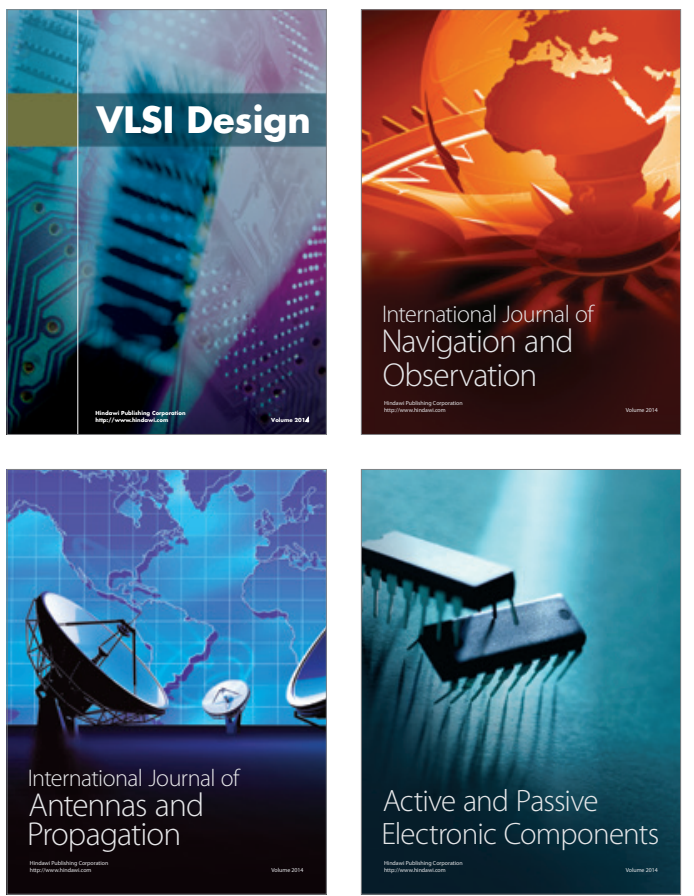
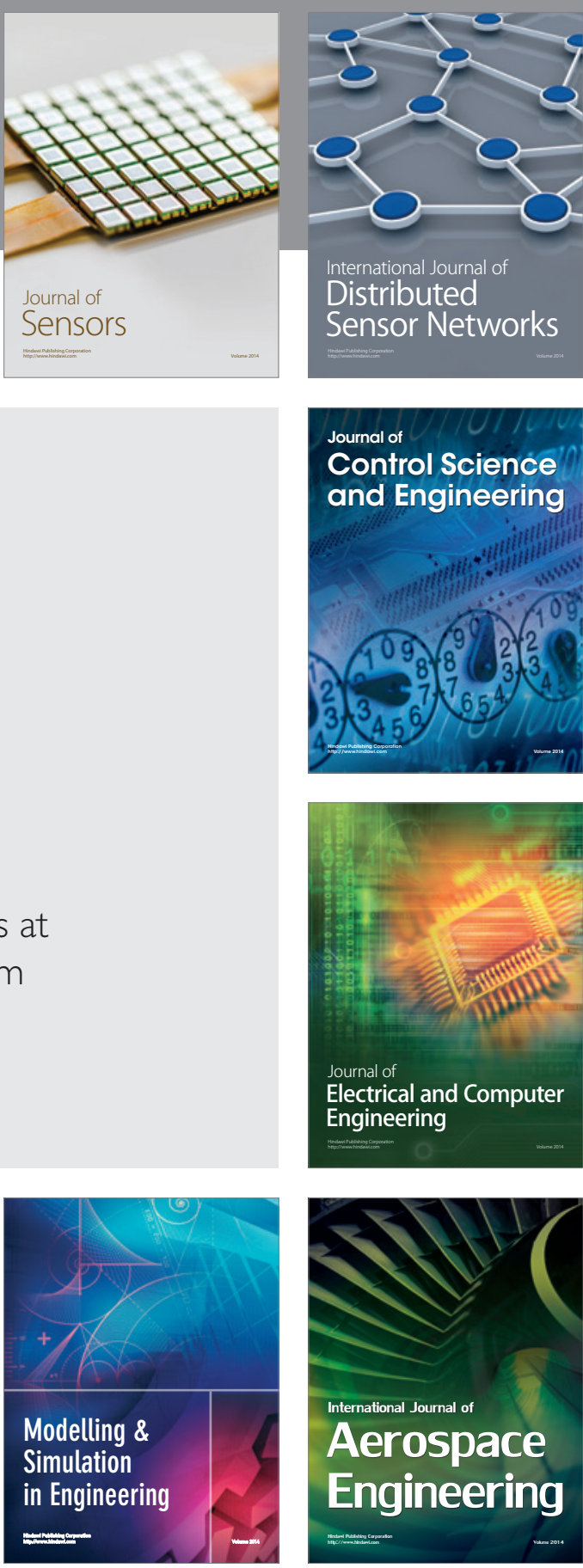

International Journal of

Distributed

Sensor Networks

Journal of

Control Science

and Engineering
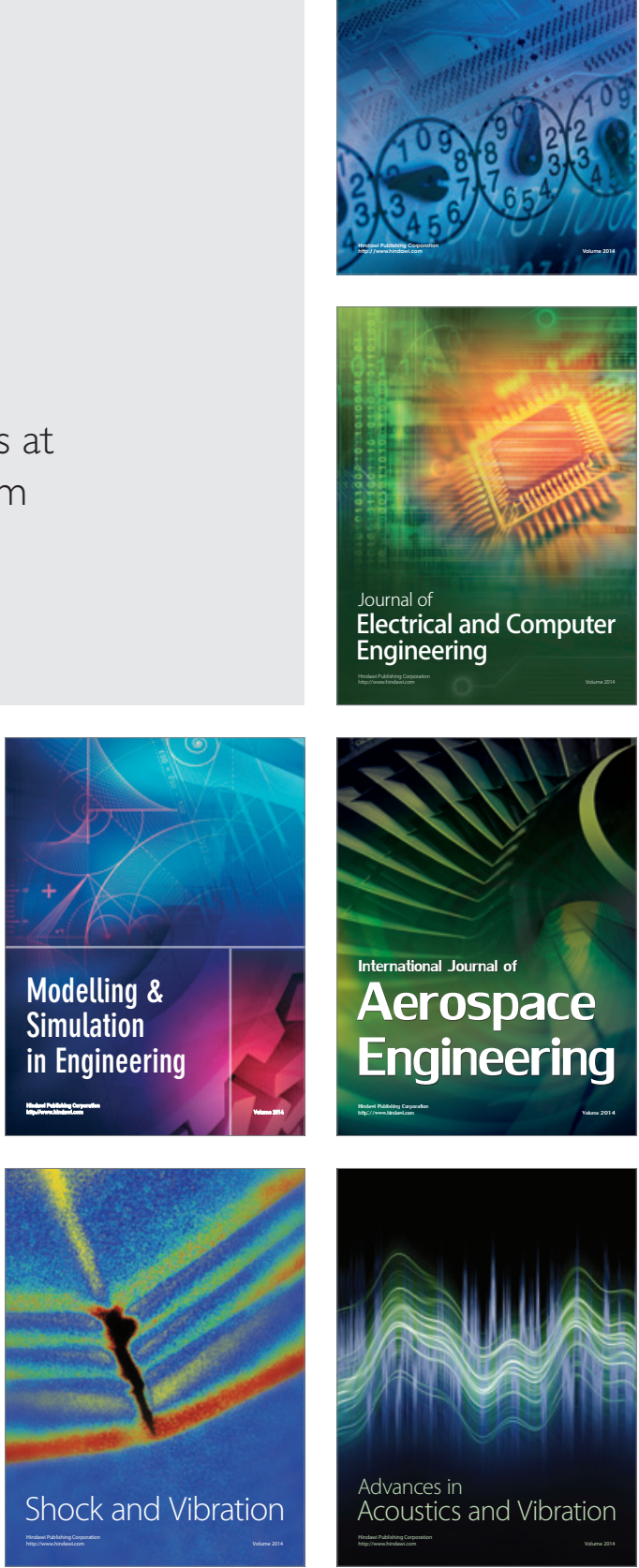